\title{
“SAY NINETYNINE”: IT’S NEVER TOO LATE TO RECOVER FROM COVID-19
}

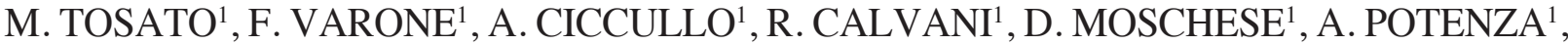 \\ M. SICILIANO ${ }^{1}$, M. FANTONI ${ }^{1}$
}

\author{
1. Fondazione Policlinico Universitario Agostino Gemelli IRCSS, Rome, Italy; ORCID: https://orcid.org/0000-0001-5750-9746. \\ Corresponding author: Matteo Tosato, Fondazione Policlinico Universitario Agostino Gemelli IRCSS, Rome, Italy, email: matteo.tosato@policlinicogemelli.it
}

\begin{abstract}
COVID-19, the disease caused by severe acute respiratory syndrome coronavirus 2 (SARS-CoV-2) infection, showed higher severity and lethality in male older adults. There are currently no specific treatments. Studies are evaluating the efficacy of monoclonal antibodies against interleukin-6 receptor. Here we present the case of a 98-years old man admitted to our COVID-Hospital with acute respiratory failure. Comprehensive geriatric assessment showed no signs of frailty. First-line therapy with hydroxychloroquine and anticoagulants was not effective. Patient was administered intravenous monoclonal antibodies, and he showed remarkable clinical improvement. This case suggests that age alone should not preclude access to new therapeutic approaches. Comprehensive, multisciplinary, multidomain approaches are needed to develop patient-tailored treatments against COVID-19.
\end{abstract}

Key words: Frailty, covid-19, comprehensive geriatric assesment, aging.

\section{Introduction}

In late 2019, a novel betacoronavirus (SARS-CoV-2), emerged in the province of Hubei, China. The disease related to SARS-CoV-2, named COVID-19, rapidly spread all over the world and was declared a global pandemic by the World Health Organization (WHO). While the majority of cases present with no or mild respiratory symptoms, about $20 \%$ of the cases could develop severe forms of the disease, with life-threatening complications such as respiratory failure and multiorgan dysfunction (1). Fatality rates vary between studies, but large cohort observational studies reported a greater disease severity in older patients (1-2).

We have recently observed a demographic shift towards older ages in COVID-19 patients admitted to our COVID Hospital. This phenomenon was partly due to newly discovered outbreaks in nursing homes and rehabilitation units. Older patients present specific characteristics, such as frailty, multimorbidity, polypharmacotherapy, and other conditions that render their management particularly difficult. Their unmet clinical needs represent the "new challenge" in COVID-19 pandemic.

Hereby we report a case of severe COVID-19 pneumonia in an old man admitted to our COVID-hospital in Rome, Italy.

\section{Case representation}

A 98-years old man was admitted to our hospital on April 15th, following a 2-day history of cough and dyspnea. The patient came from a rehab-unit where he was admitted following hip fracture. He was independent in daily activities before hospital admission and not frail (Clinical Frailty Scale score was 3) (3). Chronic obstructive pulmonary disease, hypertension, chronic lymphocytosis and benign prostatic hypertrophy were also present in his medical history. Blood tests at admission showed C-Reactive Protein $221.7 \mathrm{mg} / \mathrm{L}$ (Reference Value [RV] <5.0), D-dimer $4425 \mathrm{ng} / \mathrm{mL}(\mathrm{RV}$ <500), ferritin $1286 \mathrm{ng} / \mathrm{mL}$ (RV 21-275), IL-6 $105.8 \mathrm{ng} / \mathrm{L}$ $(\mathrm{RV}<4.4)$. Chest X-rays documented signs of interstitial pneumonia (Figure 1). Patient presented in severe clinical conditions, hypoxemic, requiring oxygen supplementation with a $\mathrm{FiO} 2$ of 0.6 . Patient was treated immediately with hydroxychloroquine and anticoagulant therapy with no clinical benefits. At day 5 , he was administered intravenous sarilumab (400 $\mathrm{mg}$ as single dose). The day after, patient started showing remarkable clinical improvement. Over the following weeks, we observed a full consciousness recovery and improvement in respiratory function, with progressive reduction of oxygen supplementation. On May 8th he was discharged from the hospital after blowing out his 99 candles.

Figure 1

Patient's chest $\mathrm{X}$-rays at hospital admission

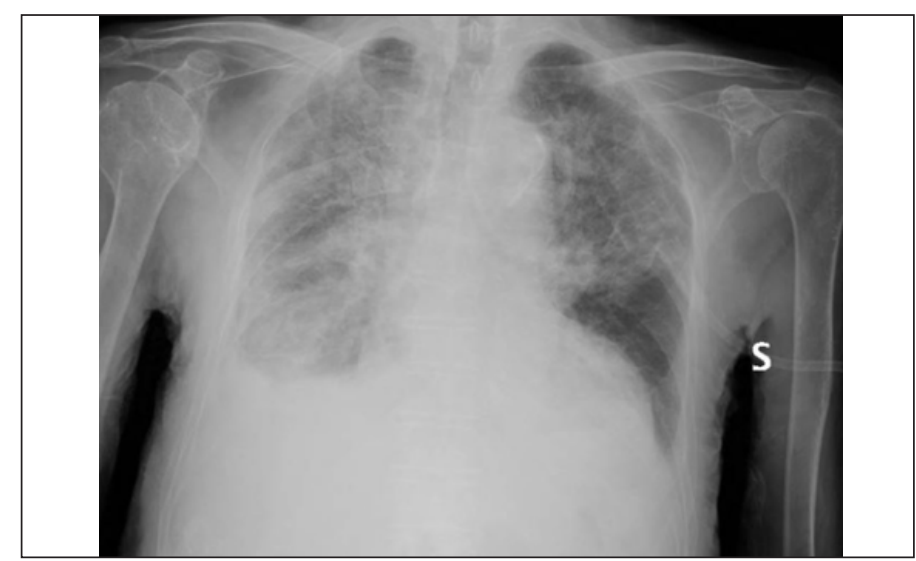




\section{THE JOURNAL OF FRAILTY \& AGING}

\section{Discussion}

As of 9 May 2020, the current COVID-19 pandemic has already caused over 270,000 deaths worldwide (4). Older patients are more vulnerable to COVID-19 as witnessed by their higher hospitalization and mortality rate; in particular, institutionalized older persons appear particularly at risk of experiencing negative outcomes. COVID-19 has now become the main challenge in geriatric care. Thus, geriatricians are increasingly recognized as key figures in multidisciplinary hospital teams dealing with the COVID-19 pandemic (5). There are currently no specific treatments available for COVID19. Several studies are underway to evaluate the efficacy of monoclonal antibodies against the interleukin- 6 receptor (tocilizumab and sarilumab) in mitigating the cytokine cascade and improving the clinical course of the disease. However, older adults are usually excluded from clinical trials and therefore are at risk, during this pandemic phase, of not having access to the treatments being studied. In our case, a 98-years old patient optimally responded to off-label sarilumab with marked improvement in clinical conditions and no adverse reactions reported. Although clinical trials will be necessary to assess safety and efficacy of sarilumab in the treatment of COVID-19, our finding may be promising.

\section{Conclusion}

This case suggests that age alone should not preclude access to new therapeutic approaches. Comprehensive, multisciplinary, multidomain approaches assessing, among others, comorbidity burden and frailty status, are needed to develop patient-tailored treatments against COVID-19.

Funding: This case report received no specific grant from any funding agency in the public, commercial, or not-for-profit sectors.

Conflict of interest: On behalf of all authors, the corresponding author states that there is no conflict of interest.

Ethical approval: Not applicable. No research study involved.

Statement of human and animal rights: All procedures performed in the study were in accordance with the ethical standards of the institutional or national research committee and with the 1964 Helsinki declaration and its later amendments or comparable ethical standards and conformed to the Declaration of Helsinki on human research.

Informed consent: The patient included in the study gave written informed consent for the publication.

\section{References}

1. Wu Z, McGoogan JM. Characteristics of and Important Lessons From the Coronavirus Disease 2019 (COVID-19) Outbreak in China: Summary of a Report of 72314 Cases From the Chinese Center for Disease Control and Prevention [published online ahead of print, 2020 Feb 24]. JAMA. 2020;10.1001/jama.2020.2648.

2. Chen R, Liang W, Jiang M, et al. Risk Factors of Fatal Outcome in Hospitalized Subjects With Coronavirus Disease 2019 From a Nationwide Analysis in China [published online ahead of print, 2020 Apr 15]. Chest. 2020;S0012-3692(20)30710-8. doi:10.1016/j.chest.2020.04.010

3. Rockwood K, Song X, MacKnight C, Bergman H, et al. A global clinical measure of fitness and frailty in elderly people. CMAJ. 2005 Aug 30;173(5):489-95.

4. John Hopkins University - Coronavirus resource center. Accessed on May 9th. Available at https://coronavirus.jhu.edu/

5. Landi F, Barillaro C, Bellieni A, et al. The New Challenge of Geriatrics: Saving Frail Older People from the SARS-COV-2 Pandemic Infection. J Nutr Health Aging. 2020;24(5):466-470. doi:10.1007/s12603-020-1356-x 